\title{
Proceso de selección de los coordinadores de nivel de una institución educativa
}

\author{
Selection process of level coordinators of an educational \\ institution
}

\section{• JENNY JOSEFINA GUERRA}

jennyplacencia10@gmail.com

Código ORCID: 0000-0002-1829-6159

Escuela Técnica Agropecuaria El Tigre, estado Anzoátegui, Venezuela

\begin{abstract}
Resumen El presente trabajo tuvo como objetivo analizar el proceso empleado para la selección de los coordinadores de nivel de una institución educativa. La investigación fue de tipo cuantitativo, con un diseño de investigación de campo en un nivel descriptivo. La muestra estuvo constituida por dos directivos, diez docentes coordinadores de nivel, dos coordinadores de Control de Estudios y ocho docentes de aula de la Unidad Educativa Nacional "Alberto Isaac Padra" del estado Guárico. La técnica fue una encuesta, los instrumentos un cuestionario y una escala tipo Likert. Los resultados arrojaron que el proceso de selección no se cumple en concordancia con lo establecido en el reglamento respectivo, pues en esta institución se hace a través de una elección por votación a mano alzada realizada por los docentes de aula, por lo que se invita a hacer los ajustes en atención a lo establecido en el marco legal.
\end{abstract}

Palabras clave: Docentes coordinadores de nivel; funciones de los coordinadores; proceso de selección

\begin{abstract}
The objective of this work was to analyze the process used to select the level coordinators of an educational institution. The research was quantitative, with a descriptive level field research design. The sample consisted of two directors, ten coordinating teachers at the level, two coordinators of Study Control and eight classroom teachers from the "Alberto Isaac Padra" National Educational Unit in the state of Guárico. The technique was a survey, the instruments a questionnaire and a Likert-type scale. The results showed that the selection process is not fulfilled in accordance with the provisions of the respective regulations, since in this institution it is done through an election by a show of hands by the classroom teachers, for which it is invited to make adjustments in accordance with the provisions of the legal framework
\end{abstract}

Keywords: Level coordinator teachers; coordinator functions; selection process 


\section{INTRODUCCIÓN}

La evaluación ha pasado por un arduo proceso de definiciones y redefiniciones. Esto ha originado que sus seguidores comiencen a clasificarla de diferentes maneras. Una de esas clasificaciones es la evaluación institucional. Para Estaba (1996) la evaluación institucional es "El proceso de juzgar tanto los resultados como los procesos de un sistema en operación para cumplir con tres tareas básicas: controlar la marcha del sistema, conocer los resultados actuales, y orientar la política institucional" ( $p$. 37). Este autor enfatiza la necesidad de examinar constantemente los objetivos propuestos por la institución, para advertir sobre los resultados actuales y encaminar sus metas.

Actualmente, en Venezuela la evaluación institucional está planificada por el Ministerio de Educación Universitaria que busca por la vía de la evaluación, establecer mecanismos de control, de gestión y financieros, cuyo fin es estar engranados a los retos trazados por el gobierno nacional para lograr el nuevo modelo de desarrollo económico y social del país. Frente a este panorama, la evaluación institucional es un proceso propuesto, planificado y organizado por el ente rector encargado de dirigir las políticas educativas de una nación. En el caso de Venezuela en ente rector para el subsistema de educación básica es el Ministerio del Poder Popular para la Educación quien se ocupa de diseñar, coordinar y ejecutar este tipo de evaluación a fin de lograr el fortalecimiento y la calidad de las instituciones educativas.

De acuerdo con lo descrito anteriormente, se considera que la evaluación institucional abarca de manera integral y completa el análisis del funcionamiento de una institución ya sea de índole educativa o no. Por tanto, la presente investigación se enmarca dentro de la evaluación del desempeño o de la gestión de las tareas de carácter administrativo de los coordinadores del nivel de Educación Media General en la Unidad Educativa "Alberto Isaac Padra". Al respecto Alles (2008) establece:

Las evaluaciones de desempeño son útiles y necesarias para: (a) tomar decisiones de promociones y remuneraciones, (b) reunir y revisar las evaluaciones de los jefes y subordinados sobre el comportamiento del empleado en relación con el trabajo... Una evaluación del desempeño debe realizarse siempre con relación a lo requerido por el puesto. Solo se podrá decir que una persona se desempeña bien o mal en relación con algo, en este caso, "ese algo" es el puesto que ocupa. (p. 31)

Aunado a la cita anterior, los individuos, en el desempeño de sus funciones, adoptan conductas que le son propias en diferentes situaciones y ambientes, lo que les da una característica muy particular en su forma de realizarse.

Cabe destacar que la evaluación del desempeño surgió en los Estados Unidos de Norteamérica en el decenio de 1920 a 1930, cuando las empresas comenzaron a introducir políticas retributivas al personal, las mismas estaban relacionadas con las responsabilidades del puesto de trabajo y con los aportes de los empleados al éxito de la organización. Autores como Robalino y Corner (2007) plantean que en América Latina, el personal docente debe someterse consciente y periódicamente a un 
proceso de evaluación de su desempeño, por cuanto se requiere de un continuo perfeccionamiento. (p. 97)

Por otro lado, Valdés (2000) ha analizado el hecho de que algunos agentes educativos obstaculizan todo esfuerzo porque se instauren políticas de mejoras en sus sistemas educativos, a partir de posiciones básicamente gremiales, las cuales tratando de "proteger al docente", olvidan el derecho de los estudiantes a recibir una educación cualitativamente superior e incluso, no reflexionan en el derecho que tienen los docentes a recibir acciones de asesoramiento y control que contribuyan al mejoramiento de su trabajo. (p. 43)

Por lo tanto, el desempeño docente se refiere pues al cumplimiento de ciertas actividades y del deber. Dicho desempeño está regulado por la Constitución de la República Bolivariana de Venezuela (2000), Ley Orgánica de Educación (2009) y el Reglamento del Ejercicio de la Profesión Docente (2000); explícitas leyes que establecen las funciones a cumplir como valor de formadores de las generaciones emergentes. Para Valdés (2000), la evaluación del docente juega un papel de primer orden, pues permite caracterizar su desempeño $y$, por lo tanto, propiciar su desarrollo futuro, al propio tiempo que constituye una vía fundamental para su atención y estimulación (p. 65). Así pues, la evaluación del desempeño tiene como principal objetivo determinar las cualidades profesionales, la preparación y el rendimiento del educador en su entorno laboral.

Por esta razón es posible someter a evaluación el desempeño laboral de todo docente, considerando que debe conocer cabalmente cada una de sus funciones en el ámbito dentro del cual ejerce las mismas. Para
Ordoñez (2009), los profesionales de la docencia que ejerzan el cargo de coordinadores a tiempo completo son considerados colaboradores inmediatos de la Dirección del plantel, con la cual comparten responsabilidades en la gerencia del mismo (p. 23). Los docentes coordinadores de nivel son entes operativos que se desempeñan en el nivel de Educación Media General, es decir, son quienes orientan y coordinan las actividades del proceso de enseñanza y aprendizaje de primero a quinto año, considerando un coordinador para cada año de escolaridad, por cada cinco secciones.

Por otro lado, el coordinador a tiempo completo debe reunir un perfil ocupacional necesario para optimizar su desempeño laboral, como el conocimiento de políticas y filosofías educativas que permitan orientar y asesorar a los estudiantes en el cumplimiento de sus deberes y derechos. Así como administrar de manera eficiente lo relacionado con la seccional que coordina, con la aplicación de leyes, reglamentos, resoluciones y decretos. (Ordoñez, 2009, p. 24)

Debido a la función social que realizan estos docentes coordinadores, constantemente están sometidos a una valoración de todos los que reciben directa $o$ indirectamente sus servicios. Por lo que se considera que estas valoraciones y opiniones que se producen de forma espontánea, sobre su comportamiento o competencia pueden dar lugar a situaciones de ambigüedad, a contradicciones, a un alto nivel de subjetivismo $y$, en ocasiones, pueden ser causa de decisiones inadecuadas, de insatisfacción y desmotivación de los docentes.

En relación a esta situación, la evaluación del profesorado puede servir para dos propósitos básicos: responsabilidad y desarrollo profesional. 
El primero de ellos implica la reunión de datos para determinar el grado en que los profesores han alcanzado niveles mínimos aceptables de competencia y definido los estándares que deben lograr. El interés por la responsabilidad ha tendido a dominar los pensamientos y las acciones de los administradores responsables de la evaluación de los profesores. El segundo propósito referido al desarrollo profesional, ha ido aumentando en años recientes y tiene como foco central de atención, la reunión de datos para ayudar a progresar a aquellos profesores que son, por lo menos, mínimamente competentes en su trabajo (Stiggins y Duke, 2008 , p. 109). Por consiguiente, se refiere una recopilación de información que permite hacer reflexiones respecto al docente en una coordinación

Para efectos de la presente investigación, se consideró la evaluación del desempeño profesional desde el rol de docente coordinador de nivel, conceptualizando el desempeño docente como:

Un proceso sistemático de
obtención de datos válidos y
fiables, con el objetivo de
comprobar y valorar el efecto
educativo que produce en los
alumnos el despliegue de sus
capacidades pedagógicas, su
emocionalidad, responsabilidad
laboral y la naturaleza de sus
relaciones interpersonales con
los alumnos, padres, directivos,
colegas y representantes de las
instituciones de la comunidad.
(Valdés, 2000. p. 54)

De este modo, para la evaluación del desempeño profesional en el presente estudio, se analizaron las competencias en relación con el nivel requerido para el puesto de trabajo, pues la finalidad de esta evaluación fue identificar y ponderar comportamientos laborales en relación con el puesto de trabajo. De allí que el propósito de este trabajo se enfocara en analizar el proceso empleado para la selección de los coordinadores de nivel de la Unidad Educativa Nacional "Alberto Isaac Padra" (Guerra, 2014).

En cuanto a un proceso evaluativo dentro de esta institución, se ha observado que se realiza evaluación solo al personal docente interino con funciones de aula, valoración que es llevada a cabo para ingresar como personal titular ante el Ministerio del Poder Popular para la Educación. Sin embargo, no ocurre una valoración sistemática en la institución una vez que el docente evaluado ingresa.

\section{Evaluación del desempeño docente}

Las organizaciones educativas no han escapado a la necesidad de evaluar sus instituciones y su personal: esto lleva a identificar el desempeño profesional del docente como un aspecto importante para alcanzar una exitosa gestión. A efectos de esta investigación, se asumió la evaluación de desempeño docente según la función que realiza este profesional. En concordancia con Valdés (2000) La evaluación del desempeño del docente es un proceso inminente dentro de la evaluación institucional, a través de la misma se asigna valor al curso de una acción. En este sentido, es necesario precisar las funciones que debería cumplir la evaluación del desempeño docente. A respecto, Valdés (2000) establece las siguientes: Función de Diagnóstico, de la cual se derivan acciones de capacitación y superación que contribuyan a la 
corrección de imperfecciones; Función Instructiva de la cual derivan indicadores del desempeño del docente, la Función Educativa, donde se toma en cuenta las motivaciones y actitudes de los docentes hacia su trabajo y la Función Desarrolladora en la cual el docente es capaz de autoevaluarse para mejorar sus habilidades.

Para la evaluación del desempeño docente, en esta investigación, se analizaron las competencias en relación con el nivel requerido para el cargo de coordinador de nivel, los cuales son los profesionales de la docencia que laboran en el Nivel de Educación Media General correspondiente al subsistema de educación básica y que asumen la función de ser jefes de seccionales. Por tanto, son los encargados de colaborar directamente con la Dirección del Plantel en la organización y ejecución de las acciones de gerencia. Sus actividades también giran en torno a la orientación, planificación y coordinación del desarrollo de los procesos de enseñanza y aprendizaje conjuntamente con los docentes de aula. (Ordoñez, 2009, p. 67).

Este autor establece que los coordinadores deben ejecutar cuatro grandes funciones: Organización, donde determinan qué acciones o tareas deben llevarse a cabo; Planificación, orientada a definir las metas de la organización y las estrategias para alcanzarlas; Dirección y supervisión, relacionada con el monitoreo del desempeño de los empleados de la organización y la Administración donde se motivar a los empleados y se rigen las actividades de los demás mediante el empleo de los mejores canales de comunicación.

\section{MÉTODO}

Según la naturaleza del estudio y los objetivos planteados, la presente investigación estuvo enmarcada dentro del enfoque cuantitativo. Los datos recogidos se contabilizaron a fin de evaluar el desempeño de los coordinadores de nivel en la Unidad Educativa Nacional "Alberto Isaac Padra" El Sombrero, ubicada en el Municipio Mellado, Estado Guárico durante el año escolar 20122013.

Tomando en cuenta las características de la investigación, el presente estudio se desarrolló bajo la modalidad de investigación de campo. Los datos se obtuvieron directamente de la realidad, es decir, en la institución. A su v ez, este trabajo responde a un carácter descriptivo, por cuanto se registró la información ordenadamente del hecho investigado para tener las herramientas necesarias a fin de evaluar el desempeño de los coordinadores de nivel en el plantel.

En tal sentido y a efectos de la presente investigación el universo de estudio estuvo conformado por el personal directivo, por todos los docentes coordinadores y por los docentes de aula que laboran en esta institución. Es decir, por un total de 62 miembros del personal docente. Para Hernández, Fernández y Baptista (2004), la población o universo es el conjunto de todos los casos que concuerdan con una serie de especificaciones (p. 302). En cuanto a la muestra, se extrajo a un grupo de la población ajustado a ciertos criterios de selección. Hernández, Fernández y Baptista (2004) la definen como "Un subgrupo de la población del cual se recolectan los datos y debe ser representativo de dicha población" (p. 302). Así la muestra quedó conformada por: 2 directivos (1 por cada planta física de la institución), 10 docentes coordinadores de nivel que laboran en la institución, 2 docentes coordinadores de Control 
de Estudios (1 por cada planta física), y 8 docentes de aula elegidos por los 4 coordinadores de nivel.

La técnica utilizada fue la encuesta que se aplicó a toda la muestra objeto de estudio. Los instrumentos empleados para la recolección de los datos fueron: un cuestionario de preguntas cerradas y dicotómicas que estuvo dirigido solo al personal directivo. El segundo instrumento fue una escala tipo Likert dirigido a los docentes coordinadores de nivel. La investigación se desarrolló en dos etapas: análisis del proceso de selección y diagnóstico a los coordinadores las cuales se detallan a continuación.

En la etapa de análisis del proceso de selección se procedió a elaborar una primera versión del cuestionario que luego fue validado por expertos. Después de la validación y aplicación de prueba pilota a dos subdirectores de la institución, se diseñó la versión final del instrumento. Seguidamente se contactó al personal directivo de la Unidad Educativa Nacional "Alberto Isaac Padra" para solicitarles el llenado a fin de analizar el proceso empleado para la selección de los coordinadores de nivel en esta institución. La finalidad de este instrumento fue recoger información directa de los actores encargados del proceso de selección para los docentes coordinadores de nivel en la $U$. E. N. "Alberto Isaac Padra" $y$, finalmente, analizar cómo se ejecuta dicho proceso en esta institución.

En la etapa dos de diagnóstico a los coordinadores se elaboró una escala tipo Likert que luego de su validación por parte de expertos, se contactó a los docentes coordinadores de nivel para aplicarles el instrumento a fin de describir el grado de conocimiento que poseen estos acerca de las funciones inherentes a su cargo en la Unidad Educativa Nacional "Alberto Isaac Padra".

De este modo, según lo observado se pudo determinar que el $80 \%$ de los indicadores no se cumplen en el proceso de selección de los docentes coordinadores de nivel en la U. E. N. "Alberto Isaac Padra", mientras que el $20 \%$ de ellos sí se realizan. Más concretamente, para designar a un docente coordinador de nivel en esta institución solo se evalúan las credenciales de los aspirantes y se informa a los interesados una vez tomada la decisión. El resto de los indicadores son obviados totalmente; en consecuencia, no se realiza una convocatoria pública, no se lleva a cabo un proceso formal de inscripción de los aspirantes, no se constituye formalmente el jurado tal como lo establece el Reglamento del Ejercicio de la Profesión Docente, no se aplican pruebas de oposición ni se publica resultado alguno una vez tomada la decisión.

\section{RESULTADOS Y DISCUSIÓN}

Luego de que se aplicaron los instrumentos de recolección de datos, los resultados fueron procesados a través de la clasificación, ordenación y tabulación de estos. Se empleó la estadística descriptiva a través del análisis porcentual y las frecuencias absolutas como técnica de análisis de los datos, la cual es definida por Hurtado (2008), como aquella que: "Puede ser utilizada para el análisis en investigaciones descriptivas cuando el interés del investigador está en conocer la magnitud o intensidad con la cual se presenta un evento..." (p. 523). Así pues, una vez obtenidas las frecuencias de cada ítem, se procedió a tabular los datos en cuadros y se representaron mediante porcentajes a través de gráficos de 
diagramas de barra. Esto permitió hacer un análisis para luego realizar las conclusiones y recomendaciones.

En lo referente al cuestionario aplicado a los directivos, estos expresaron que, desde hace más de 10 años, la elección de los docentes coordinadores de nivel no se realiza para optar a la titularidad. Los coordinadores actuales son interinos los cuales son elegidos por los docentes de aula mediante un colectivo y por votación a mano alzada. Luego el director lleva la propuesta ante el municipio escolar y este es quien emite la última palabra. Todo esto sucede sin la aplicación de una preparación previa de adiestramiento que dé paso al cumplimiento de las funciones de estos. Llama la atención que quienes eligen a los coordinadores de nivel son los mismos docentes de aula los cuales posteriormente se convierten en los subordinados de estos coordinadores; lo que se aleja totalmente de lo establecido en la ley, en el artículo 68:

Los jurados examinadores estarán integrados por tres miembros (3) principales y tres (3) miembros suplentes propuestos por la Junta Calificadora Nacional, Zonal, Estadal o Municipal... Los integrantes del jurado no deben estar vinculados a los aspirantes por lazos de parentesco hasta el cuarto grado de consanguineidad y segundo de afinidad, ni por relación matrimonial. (Reglamento del Ejercicio de la Profesión Docente, 2000, p. 47)

El análisis anterior explica que en la U. E. N. "Alberto Isaac Padra" no se ejecuta debidamente el proceso para la selección de los docentes coordinadores de nivel establecido en la normativa legal. Para algunos autores como Alles
(2008) el no hacer un proceso apropiado de selección del personal no contribuye con el aprovechamiento adecuado de los recursos humanos. Explica esta autora que algunas veces se asignan cargos solo para incrementar el salario o por algún parentesco asociado a quienes deben tomar las decisiones; por tanto, las consecuencias negativas o no de este tipo de decisiones se observan en el desempeño de las funciones del personal.

Más concretamente, para designar a un docente coordinador de nivel en esta institución solo se evalúan las credenciales de los aspirantes y se informa a los interesados una vez tomada la decisión. El resto de los indicadores son obviados totalmente; en consecuencia, no se realiza una convocatoria pública, no se lleva a cabo un proceso formal de inscripción de los aspirantes, no se constituye formalmente el jurado tal como lo establece el Reglamento del Ejercicio de la Profesión Docente, no se aplican pruebas de oposición ni se publica resultado alguno una vez tomada la decisión

Luego de aplicada la encuesta los coordinadores de procesaron los datos obtenidos a través de una matriz de datos $A$ efectos del análisis, se asignó a cada coordinador un número para mantener el anonimato de su identificación. En la matriz de datos se evidencian las respuestas dadas por cada coordinador en la escala tipo Likert aplicada para saber el nivel de conocimiento que poseen sobre sus funciones administrativas. Además, esta incluye: Ítems referidos a las funciones de los docentes coordinadores de nivel y la puntuación obtenida por cada respondiente según la escala tipo Likert. A cada una de las opciones de respuesta en la escala se le otorgó una puntuación a saber: Siempre (5 puntos), casi siempre (4 puntos) 
algunas veces (3), casi nunca (2 puntos) y nunca (1 punto). La puntuación máxima corresponde a un total de 120 puntos y la mínima, 24 puntos. A continuación, se desglosa el análisis por individual de cada ítem.

En la primera proposición (ver Tabla 1) dirigida a saber si los docentes coordinadores conocen la función referida a la organización de expedientes estudiantiles al inicio del año escolar, el $70 \%$ de la muestra respondió que casi siempre lo realizan, mientras que el $30 \%$ restante afirmó que siempre lo hace. Dejando claro que los docentes coordinadores en general reconocen dicha función.

Tabla 1. Distribución de frecuencia ítem 1

\begin{tabular}{lcc}
\hline Organiza expedientes & $\mathrm{fi}$ & $\%$ \\
\hline Siempre & 3 & $30 \%$ \\
Casi siempre & 7 & $70 \%$ \\
Algunas veces & 0 & 0 \\
Casi nunca & 0 & 0 \\
Nunca & 0 & 0 \\
Totales & $\mathbf{1 0}$ & $\mathbf{1 0 0 \%}$ \\
\hline
\end{tabular}

En la Tabla 2 los datos obtenidos demuestran que solo el $60 \%$ de los docentes coordinadores de nivel conoce la función de convocar a reuniones porque el $20 \%$ respondió siempre y un $40 \%$ respondió casi siempre. Sin embargo, existe otro $40 \%$ de estos docentes que pareciera no reconocer entre sus funciones hacer tales convocatorias con frecuencia.

Tabla 2. Distribución de frecuencia ítem 2

\begin{tabular}{lcc}
\hline Convoca a reuniones & $\mathrm{fi}$ & $\%$ \\
\hline Siempre & 2 & $20 \%$ \\
Casi siempre & 4 & $40 \%$ \\
Algunas veces & 4 & $40 \%$ \\
Casi nunca & 0 & 0 \\
Nunca & 0 & 0 \\
Totales & $\mathbf{1 0}$ & $\mathbf{1 0 0 \%}$ \\
\hline
\end{tabular}

En la Tabla 3, relacionado con la función de llevar control de asistencia diaria de los estudiantes por asignatura, el $90 \%$ de los docentes coordinadores de nivel reconoce realizar siempre o casi siempre esta función; solo un 10\% demostró que casi nunca lo hacen. 
Tabla 3. Distribución de frecuencia ítem 3

\begin{tabular}{lcc}
\hline Lleva control de asistencia estudiantil & $\mathbf{f i}$ & $\mathbf{\%}$ \\
\hline Siempre & 8 & $80 \%$ \\
Casi siempre & 1 & $10 \%$ \\
Algunas veces & 0 & 0 \\
Casi nunca & 1 & $10 \%$ \\
Nunca & 0 & 0 \\
Totales & $\mathbf{1 0}$ & $\mathbf{1 0 0 \%}$ \\
\hline
\end{tabular}

En la Tabla 4, el 100\% de los docentes coordinadores de nivel entregan mensualmente la asistencia de los docentes de aula adscritos a la seccional, pues sus respuestas apuntaron hacia siempre (en un $90 \%$ ) y casi siempre (en un 10\%).

Tabla 4. Distribución de frecuencia ítem 4

\begin{tabular}{lcc}
\hline Entrega control de asistencia de los docentes & $\mathbf{f i}$ & \% \\
\hline Siempre & 9 & $90 \%$ \\
Casi siempre & 1 & $10 \%$ \\
Algunas veces & 0 & 0 \\
Casi nunca & 0 & 0 \\
Nunca & 0 & 0 \\
Totales & $\mathbf{1 0}$ & $\mathbf{1 0 0 \%}$ \\
\hline
\end{tabular}

En la Tabla 5 el $80 \%$ de los docentes encuestados reconoce la función de elaborar carteleras en una frecuencia de siempre y casi siempre, solo el 10\% manifiesta realizarla algunas veces y otro $10 \%$ nunca la ejecuta; es decir, hay un $20 \%$ que desconoce esta función.

Tabla 5. Distribución de frecuencia ítem 5

\begin{tabular}{lcc}
\hline Elabora carteleras informativas & $\mathbf{f i}$ & $\mathbf{\%}$ \\
\hline Siempre & 5 & $50 \%$ \\
Casi siempre & 3 & $30 \%$ \\
Algunas veces & 1 & $10 \%$ \\
Casi nunca & 0 & 0 \\
Nunca & 1 & $10 \%$ \\
Totales & $\mathbf{1 0}$ & $\mathbf{1 0 0 \%}$ \\
\hline
\end{tabular}

En la Tabla 6, el 90\% de la muestra indicó reconocer la función de asignar responsabilidades a los docentes guías; solo el $10 \%$ algunas veces lo realiza; es decir, un bajo porcentaje desconoce esta función. 
Tabla 6. Distribución de Frecuencia ítem 6

\begin{tabular}{lcc}
\hline Asigna responsabilidad a los docentes guías & $\mathbf{f i}$ & $\%$ \\
\hline Siempre & 9 & $90 \%$ \\
Casi siempre & 0 & 0 \\
Algunas veces & 1 & $10 \%$ \\
Casi nunca & 0 & 0 \\
Nunca & 0 & 0 \\
Totales & $\mathbf{1 0}$ & $\mathbf{1 0 0 \%}$ \\
\hline
\end{tabular}

En la Tabla 7 el 100\% de la muestra identificó la función de organizar matrícula y listado de estudiantes al comienzo del año escolar; es decir, el 90\% manifestó que siempre ejecutan la actividad y el $10 \%$ casi siempre.

Tabla 7. Distribución de Frecuencia ítem 7

\begin{tabular}{lcc}
\hline Organiza matrícula inicial y listado de estudiantes & $\mathbf{f i}$ & $\mathbf{\%}$ \\
\hline Siempre & 9 & $90 \%$ \\
Casi siempre & 1 & $10 \%$ \\
Algunas veces & 0 & 0 \\
Casi nunca & 0 & 0 \\
Nunca & 0 & 0 \\
Totales & $\mathbf{1 0}$ & $\mathbf{1 0 0 \%}$ \\
\hline
\end{tabular}

En la Tabla 8 el $60 \%$ de los docentes coordinadores de nivel chequea necesidades de mobiliario y recursos casi siempre, pero un $40 \%$ de ellos pareciera no considerar esta actividad entre sus funciones por cuanto el $30 \%$ respondió que la realiza algunas veces y el otro $10 \%$ respondió que nunca.

Tabla 8. Distribución de Frecuencia ítem 8

\begin{tabular}{lcc}
\hline Chequea necesidades de mobiliarios y recursos & $\mathbf{f i}$ & $\mathbf{\%}$ \\
\hline Siempre & 0 & 0 \\
Casi siempre & 6 & $60 \%$ \\
Algunas veces & 3 & $30 \%$ \\
Casi nunca & 0 & 0 \\
Nunca & 1 & $10 \%$ \\
Totales & $\mathbf{1 0}$ & $\mathbf{1 0 0 \%}$ \\
\hline
\end{tabular}

En la Tabla 9 el 90\% de la muestra reconoce la función de elaborar el plan mensual y anual de la seccional, pues el $70 \%$ siempre la ejecuta y el $20 \%$ casi siempre; mientras que un $10 \%$ respondió que lo elabora algunas veces. 
Tabla 9. Distribución de frecuencia ítem 9

\begin{tabular}{lcc}
\hline Elabora plan mensual $\mathbf{y}$ anual de la seccional & $\mathbf{f i}$ & $\mathbf{\%}$ \\
\hline Siempre & 7 & $70 \%$ \\
Casi siempre & 2 & $20 \%$ \\
Algunas veces & 1 & $10 \%$ \\
Casi nunca & 0 & 0 \\
Nunca & 0 & 0 \\
Totales & $\mathbf{1 0}$ & $\mathbf{1 0 0 \%}$ \\
\hline
\end{tabular}

En la Tabla 10, el 90\% reconoce la función de elaborar calendario de actividades de la seccional, ya que el $70 \%$ respondió que siempre la ejecuta y el $20 \%$ casi siempre; solo el $10 \%$ manifestó algunas veces cumple esta función.

Tabla 10. Distribución de frecuencia ítem 10

\begin{tabular}{lcc}
\hline Elabora calendario de actividades & $\mathbf{f i}$ & $\mathbf{\%}$ \\
\hline Siempre & 7 & $70 \%$ \\
Casi siempre & 2 & $20 \%$ \\
Algunas veces & 1 & $10 \%$ \\
Casi nunca & 0 & 0 \\
Nunca & 0 & 0 \\
Totales & $\mathbf{1 0}$ & $\mathbf{1 0 0 \%}$ \\
\hline
\end{tabular}

En la Tabla 11 el 100\% de los docentes coordinadores reconocieron la función de establecer lineamientos para la seccional; es decir, el $80 \%$ respondió que siempre la ejecuta y el $20 \%$, que casi siempre la lleva a cabo. Esto demuestra que todos tienen clara esta función.

Tabla 11. Distribución de frecuencia ítem 11

\begin{tabular}{lcc}
\hline Establece lineamientos para la seccional & $\mathbf{f i}$ & $\mathbf{\%}$ \\
\hline Siempre & 8 & $80 \%$ \\
Casi siempre & 2 & $20 \%$ \\
Algunas veces & 0 & 0 \\
Casi nunca & 0 & 0 \\
Nunca & 0 & 0 \\
Totales & $\mathbf{1 0}$ & $\mathbf{1 0 0 \%}$ \\
\hline
\end{tabular}

En la Tabla 12, un $80 \%$ de la muestra reconoció la función de diseñar planes de acción para registrar las metas y estrategias que orienten los objetivos propuestos por la seccional, pues un $40 \%$ respondió que siempre la realiza y otro $40 \%$ reconoció que casi siempre la ejecuta; solo el $10 \%$ respondió que algunas veces la cumplen y otro $10 \%$ que casi nunca. Este último $20 \%$ no conoce la importancia de llevar a cabo dicha función. 
Tabla 12. Distribución de frecuencia ítem 12

\begin{tabular}{lcc}
\hline Diseña planes de acción & fr & $\%$ \\
\hline Siempre & 4 & $40 \%$ \\
Casi siempre & 4 & $40 \%$ \\
Algunas veces & 1 & $10 \%$ \\
Casi nunca & 1 & $10 \%$ \\
Nunca & 0 & 0 \\
Totales & $\mathbf{1 0}$ & $\mathbf{1 0 0 \%}$ \\
\hline
\end{tabular}

En la Tabla 13 el 50\% destacó que conoce la función de entregar informes trimestrales, porque el $30 \%$ respondió que siempre lo hace, mientras que un $20 \%$ respondió que casi siempre lo hace; pero la mitad de los docentes coordinadores de nivel evidenció en el otro $50 \%$ que no prestan atención a esta función porque un 30\% respondió que la ejecuta algunas veces y un $20 \%$ respondió que nunca entrega informes trimestrales.

Tabla 13. Distribución de frecuencia ítem 13

\begin{tabular}{lcc}
\hline Entrega informes trimestrales & $\mathbf{f i}$ & \% \\
\hline Siempre & 3 & $30 \%$ \\
Casi siempre & 2 & $20 \%$ \\
Algunas veces & 2 & $20 \%$ \\
Casi nunca & 0 & 0 \\
Nunca & 3 & $30 \%$ \\
Totales & $\mathbf{1 0}$ & $\mathbf{1 0 0 \%}$ \\
\hline
\end{tabular}

En la Tabla 14 el 100\% de la muestra conoce la función referida a coordinar el desarrollo de planes de acción. Esta afirmación se hace porque el 70\% respondió que siempre lo hace y el $30 \%$ marcó que casi siempre lo realiza.

Tabla 14. Distribución de frecuencia ítem 14

\begin{tabular}{lcc}
\hline Coordina el desarrollo de los planes de acción & $\mathbf{f i}$ & $\%$ \\
\hline Siempre & 7 & $70 \%$ \\
Casi siempre & 3 & $30 \%$ \\
Algunas veces & 0 & 0 \\
Casi nunca & 0 & 0 \\
Nunca & 0 & 0 \\
Totales & $\mathbf{1 0}$ & $\mathbf{1 0 0 \%}$ \\
\hline
\end{tabular}

En la Tabla 15 el 100\% de los docentes coordinadores de nivel conoce la función de orientar a los docentes de aula sobre actividades de planificación, supervisión, investigación, culturales y deportivas a realizarse en el plantel. 
Tabla 15. Distribución de frecuencia ítem 15

\begin{tabular}{lcc}
\hline Orienta a los docentes sobre actividades de planificación, supervisión, etc. & $\mathrm{fi}$ & $\%$ \\
\hline Siempre & 10 & $100 \%$ \\
Casi siempre & 0 & 0 \\
Algunas veces & 0 & 0 \\
Casi nunca & 0 & 0 \\
Nunca & 0 & 0 \\
Totales & $\mathbf{1 0}$ & $\mathbf{1 0 0 \%}$
\end{tabular}

En la Tabla 16, el 100\% de la muestra conoce la función de asesorar a los docentes que tiene altos índices de estudiantes aplazados, pues el 60\% respondió que siempre lo hacen, mientras el $40 \%$ marcó la opción casi siempre. Lo que evidencia que sí conocen esta función.

Tabla 16. Distribución de frecuencia ítem 16

\begin{tabular}{lcc}
\hline Asesora al docente con alto índice de aplazados & $\mathbf{f i}$ & $\mathbf{\%}$ \\
\hline Siempre & 6 & $60 \%$ \\
Casi siempre & 4 & $40 \%$ \\
Algunas veces & 0 & 0 \\
Casi nunca & 0 & 0 \\
Nunca & 0 & 0 \\
Totales & $\mathbf{1 0}$ & $\mathbf{1 0 0 \%}$ \\
\hline
\end{tabular}

En la Tabla 17 el 100\% de los docentes coordinadores de nivel conocen la función de llevar libro de actas de situaciones tratadas en reuniones con docentes, padres y representantes; es decir, el $80 \%$ de la muestra respondió que siempre lo hacen y el $20 \%$ respondió que casi siempre la ejecutan.

Tabla 17. Distribución de frecuencia ítem 17

\begin{tabular}{lcc}
\hline Lleva libro de actas de reuniones & $\mathbf{f i}$ & $\mathbf{\%}$ \\
\hline Siempre & 8 & $80 \%$ \\
Casi siempre & 2 & $20 \%$ \\
Algunas veces & 0 & 0 \\
Casi nunca & 0 & 0 \\
Nunca & 0 & 0 \\
Totales & $\mathbf{1 0}$ & $\mathbf{1 0 0 \%}$ \\
\hline
\end{tabular}

En la Tabla 18 el 70\% de la muestra respondió que siempre asesora al docente en el cumplimiento de sus funciones, el $20 \%$ respondió que casi siempre y solo el $10 \%$ manifestó que los asesora algunas veces. Lo anterior evidencia que el $90 \%$ de los docentes coordinadores de nivel manejan esta función. 
Tabla 18. Distribución de frecuencia ítem 18

\begin{tabular}{lcc}
\hline Asesora al docente en el cumplimiento de sus funciones & $\mathbf{f i}$ & $\mathbf{\%}$ \\
\hline Siempre & 7 & $70 \%$ \\
Casi siempre & 2 & $20 \%$ \\
Algunas veces & 1 & $10 \%$ \\
Casi nunca & 0 & 0 \\
Nunca & 0 & 0 \\
Totales & $\mathbf{1 0}$ & $\mathbf{1 0 0 \%}$ \\
\hline
\end{tabular}

En la Tabla 19, según respuestas de los docentes coordinadores en un 90\% aproximadamente reconocen la función establecer contactos con padres y representantes para orientar sobre la actuación general del estudiante, ya que un $70 \%$ respondió siempre y un $20 \%$ casi siempre; sin embargo un $10 \%$ respondió que lo hace algunas veces.

Tabla 19. Distribución de frecuencia ítem 19

\begin{tabular}{lcc}
\hline Establece contacto con padres y representantes & $\mathbf{f i}$ & $\mathbf{\%}$ \\
\hline Siempre & 7 & $70 \%$ \\
Casi siempre & 2 & $20 \%$ \\
Algunas veces & 1 & $10 \%$ \\
Casi nunca & 0 & 0 \\
Nunca & 0 & 0 \\
Totales & $\mathbf{1 0}$ & $\mathbf{1 0 0 \%}$ \\
\hline
\end{tabular}

En la Tabla 20, se puede decir que los docentes coordinadores en un $80 \%$ (20\% siempre y $60 \%$ casi siempre) reconocen la función sobre analizar propuestas hechas por docentes adscritos a la seccional con la intención de mejorar la disciplina en las aulas de clase y solo el $10 \%$ de los mismos lo hace algunas veces, mientras que otro $10 \%$ nunca lo hace.

Tabla 20. Distribución de frecuencia ítem 20

\begin{tabular}{lcc}
\hline Analiza propuesta de los docentes & $\mathbf{f i}$ & $\mathbf{\%}$ \\
\hline Siempre & 2 & $20 \%$ \\
Casi siempre & 6 & $60 \%$ \\
Algunas veces & 1 & $10 \%$ \\
Casi nunca & 0 & 0 \\
Nunca & 1 & $10 \%$ \\
Totales & $\mathbf{1 0}$ & $\mathbf{1 0 0 \%}$ \\
\hline
\end{tabular}

En la Tabla 21, el resultado obtenido es que un $90 \%$ (70\% siempre y $20 \%$ casi siempre) de los docentes coordinadores reconoce la función de asignar actividades a los docentes y solo un $10 \%$, algunas veces lo identifica en las funciones. 
Tabla 21. Distribución de frecuencia ítem 21

\begin{tabular}{lcc}
\hline Asigna al docente actividades para lograr metas & $\mathbf{f i}$ & $\mathbf{\%}$ \\
\hline Siempre & 7 & $70 \%$ \\
Casi siempre & 2 & $20 \%$ \\
Algunas veces & 1 & $10 \%$ \\
Casi nunca & 0 & 0 \\
Nunca & 0 & 0 \\
Totales & $\mathbf{1 0}$ & $\mathbf{1 0 0 \%}$ \\
\hline
\end{tabular}

En la Tabla 22, los docentes coordinadores respondieron en un $90 \%$ (siempre $40 \%$ y casi siempre 50\%) que motivan al personal a colaborar con la seccional, mientras el otro $10 \%$ respondió que algunas veces, confirmando así el reconocimiento de esta función.

Tabla 22. Distribución de frecuencia ítem 22

\begin{tabular}{lcc}
\hline Motiva al personal docente para colaborar con la seccional & $\mathbf{f i}$ & $\mathbf{\%}$ \\
\hline Siempre & 4 & $40 \%$ \\
Casi siempre & 5 & $50 \%$ \\
Algunas veces & 1 & $10 \%$ \\
Casi nunca & 0 & 0 \\
Nunca & 0 & 0 \\
Totales & $\mathbf{1 0}$ & $\mathbf{1 0 0 \%}$ \\
\hline
\end{tabular}

En la Tabla 23 relacionado con la función: informa a los docentes sobre lineamientos de la dirección, el $100 \%$ de los docentes coordinadores respondió siempre, lo que conlleva a determinar el reconocimiento total de esta función.

Tabla 23. Distribución de frecuencia ítem 23

\begin{tabular}{lcc}
\hline Informa al docente sobre lineamientos de la dirección & $\mathbf{f i}$ & $\mathbf{\%}$ \\
\hline Siempre & 10 & $100 \%$ \\
Casi siempre & 0 & 0 \\
Algunas veces & 0 & 0 \\
Casi nunca & 0 & 0 \\
Nunca & 0 & 0 \\
Totales & $\mathbf{1 0}$ & $\mathbf{1 0 0 \%}$ \\
\hline
\end{tabular}

En la Tabla 24, el 100\% de la muestra promueve la participación de los docentes adscritos a la seccional en las diferentes actividades planificadas por la institución, ya que en un $70 \%$ respondió siempre y otro $30 \%$ casi siempre. 
Tabla 24. Distribución de frecuencia ítem 24

\begin{tabular}{lcc}
\hline Promueve la participación del docente en actividades de la institución & fi & \% \\
\hline Siempre & 7 & $70 \%$ \\
Casi siempre & 3 & $30 \%$ \\
Algunas veces & 0 & 0 \\
Casi nunca & 0 & 0 \\
Nunca & 0 & 0 \\
Totales & $\mathbf{1 0}$ & $\mathbf{1 0 0 \%}$ \\
\hline
\end{tabular}

Los datos explicitados y analizados en los cuadros y gráficos anteriores demuestran que los docentes coordinadores de nivel encuestados tienen un alto grado de conocimiento acerca de las funciones que deben desempeñar en el cargo, en la U. E. N. "Alberto Isaac Padra". Así lo demuestran los datos recogidos en la escala tipo Likert aplicada a esta muestra donde la mayor calificación entre ellos fue de 116 puntos de un total de 120 y la mínima puntuación fue de 95 . Las opciones de las respuestas de mayor selección apuntaron hacia las posibilidades "siempre" y "casi siempre"; rara vez se evidenció un "nunca" o "casi nunca"; en fin, este instrumento reportó que los docentes coordinadores de nivel sí conocen las funciones inherentes al cargo.

\section{CONCLUSIONES}

El proceso de selección de coordinadores de nivel no se cumple en la institución analizada, esto en concordancia con lo establecido en el Reglamento del Ejercicio de la Profesión Docente, ya que esta selección se da es a través de una elección por votación a mano alzada realizada por los docentes de aula; posteriormente, el personal directivo evalúa las credenciales de los postulantes. Finalmente, se envía el resultado al Municipio Escolar donde se toma la decisión final. Una vez designado el docente coordinador de nivel no pasa por un período entrenamiento previo para asumir dicho cargo. Es importante señalar que en el cuestionario aplicado a los directivos, se conoció que desde hace más de diez años no se realiza un concurso de docente coordinador para adquirir la titularidad del cargo en cuestión.

Por lo tanto, la investigación desarrollada permitió analizar la importancia de hacer evaluaciones constantes, continuas y actualizadas al personal que labora en las instituciones educativas, con el fin de detectar necesidades de formación, conocer las inquietudes de quienes hacen vida en ellas, encontrar al profesional más idóneo para determinado cargo, motivar a las personas en el desempeño de sus funciones e involucrarlas con la misión, visión y objetivos de tales organizaciones

En fin, la misión que la evaluación consagra a la institución abordada es configurar su liderazgo en la formación, capacitación, perfeccionamiento y actualización de docentes en el rol de coordinador, de elevada calidad personal, profesional, en la producción y difusión de conocimientos socialmente válidos, que desarrolla a través de los procesos educativos con un personal competente, participativo y comprometido con los valores que promueve la institución en el marco democrático. 
Asimismo, dentro de su visión acierta el razonamiento de manifestar sus sentidos $y$ significados institucionales en el marco de una educación pública y gratuita de excelencia, comprometida con elevar el nivel de la educación mediante la formación de docentes competentes, capaces de generar conocimientos útiles para implementar los procesos pedagógicos innovadores, administrativos, sustentables y de construcción social que impulsen la transformación de realidades susceptibles en el ámbito de una formación para la vida consagrada en los principios y valores democráticos de justicia social, integración, inclusión, solidaridad, responsabilidad, bien común y compromiso profesional.

\section{REFERENCIAS}

Alles, M. (2008). Desempeño por Competencias, Evaluación 360a. Buenos Aires: Ediciones Granica S. A

Constitución de la República Bolivariana de Venezuela. Asamblea Nacional Constituyente. (2000). Gaceta Oficial de la República de Venezuela, $N^{\circ} 36.860$ (extraordinaria), diciembre

Estaba, E. (1996). Separata de la evaluación institucional en la Universidad Central de Venezuela. En Revista Planiuc, 2(3), 35 56. Recuperado de https://biblat.unam.mx/es/buscar/separat a-de-la-evaluacion-institucional

Guerra, J. (2014). Evaluación del desempeño de los docentes coordinadores de nivel en la Unidad Educativa Nacional "Alberto Isaac Padra". El Sombrero, estado Guárico (trabajo de maestría). Universidad Pedagógica Experimental Libertador,
Instituto Pedagógico de Miranda José Manuel Siso Martínez, Venezuela

Hernández S. R., Fernández C. C., y Baptista L. P., (2004). Metodología de la Investigación. (3ra. ed.). México: McGrawHill Interamericana

Hurtado, J. (2008). Metodología de la Investigación Holística. Caracas: Fundación Sypal

Ley Orgánica de Educación. (2009). Gaceta Oficial de la República Bolivariana de Venezuela $N^{\circ}$ 5.929. Caracas-Venezuela

Ordoñez, R. (2009). Manual del Supervisor, Director y Docente. Caracas: Printer Venezuela

Pestana, F. y Véliz, G. (2010). Valoración del Educador en la evaluación de los Aprendizajes. Fondo Editorial de la Universidad Pedagógica Experimental Libertador. Caracas: FEDUPEL

Reglamento del Ejercicio de la Profesión Docente. (2000). Decreto $N^{\circ} 1.011$ de fecha 4 de Octubre de 2000, Gaceta Oficial N $N^{\circ} 5.496$ Extraordinario

Robalino, M., y Körner, A. N. T. O. N. (2007). Evaluación del desempeño y carrera profesional docente: un estudio comparado entre 50 países de América y Europa. Recuperado de http: //www. unesco.

org/new/es/education/resources/onlinematerials/publications

Stiggins, J. y Duke, M. (2008). La evaluación educativa. México: McGraw-Hill Interamericana

Valdés, H. (2000). Evaluación y desempeño del docente. Encuentro Iberoamericano sobre Evaluación del Desempeño Docente. Organización de Estados Iberoamericano para la Educación, la Ciencia. Ponencia presentada en Cuba 